\title{
Gastro-Intestinal Manifestations of Pediatric Rheumatic Diseases
}

Y.A.Soliman, W.A.S.Hassan and D.G.Elsayed

Rheumatology, Rehabilitation and physical medicine, Dept., Faculty of Medicine, Benha Univ., Benha, Egypt

E-Mail: dinagomaa88m@gmail

\begin{abstract}
Background: -hepatic manifestations in RDs are not rare, so clinicians should be aware of their existence and the fact that they may occur concomitantly or serially. Furthermore, understanding the range and prevalence of GI manifestations associated with RDs, with related autoimmune disorders and with RDs treatments is essential. Pediatric rheumatic diseases are often associated with gastrointestinal symptoms. Abdominal pain is the most frequently reported complaint. Gastrointestinal manifestations of chronic rheumatic syndromes include motility disorders of collagenosis, mixed connective tissue disease and Sjögrens syndrome associated with the risk of esophagitis due to gastro-esophageal reflux. This is in addition to the non-steroidal anti-inflammatory drug-induced gastroduodenal lesions in childhood and adolescence. However, the relevance of co-medication with steroids for the pathogenesis of these lesions is controversial. This study aimed to discuss and summarize current data on gastrointestinal manifestations encountered by rheumatologic diseases in pediatric patients. Conclusion: The importance of the gastrointestinal (GI) tract in development of autoimmunity has been increasingly appreciated in rheumatological diseases. The small intestine is the most commonly affected part of the gastrointestinal system. Abnormalities in liver function tests are very common in patients with rheumatic diseases and should be further evaluated
\end{abstract}

Key words: Gastro-Intestinal Manifestations, Rheumatic Diseases.

\section{Introduction}

Rheumatological disorders (RDs) represent a diverse group of disorders that are inherited or related to environmental factors [1]. RDs frequently affect the GI tract and gastroenterologists are often asked to evaluate patients with symptoms thought to represent an underlying or coexisting RD. GI manifestations of RDs vary based on the organ involved as well as the extent and duration of involvement. Although most manifestations of RD are non-specific and not lifethreatening, the chronicity and severity of symptoms can be debilitating and may lead to serious injury. The GI disorders accompanying RDs can be divided into two major categories: intestinal disorders and disorders of the liver, biliary tracts and pancreas [2]. Some of these GI symptoms are directly related to the $\mathrm{RD}$, while others may be sequelae of treatment or due to concomitant autoimmune diseases [3].

The importance of the gastrointestinal (GI) tract in development of autoimmunity has been increasingly appreciated in human diseases. And many studies have underlined the importance of gastrointestinal inflammation in modulating local and systemic inflammation [4].

GI tract may play a major role in the pathogenesis of rheumatoid arthritis. There are also clear cases of induction of arthritis in humans by GI pathogens. Reactive arthritis is triggered by certain pathogens such as Campylobacter, Chlamydia and Salmonella (5). Following ileo-jejunal bypass, intestinal bacterial overgrowth has been tied to a high rate of inflammatory arthritis, occurring in up to $50 \%$ of patients after this procedure. Whipple's disease is a prototypical case of inflammatory arthritis occurring in the setting of intestinal colonization by a single bacterial species in a susceptible host [6,7]. And it is hypothesized that dysbiosis, or relative change in the homeostatic balance of commensal bacteria, results in altered balance of anti- and pro-inflammatory interactions and leads to dysregulation of a local immune response. As a result of this dysregulation, local $\mathrm{T}$ cells may migrate to distant lymphatic tissue, enabling them to exert effects distant to the site of activation in the intestine [3].

Pediatric rheumatic diseases are often associated with gastrointestinal symptoms. Abdominal pain is the most frequently reported complaint. Gastrointestinal manifestations of chronic rheumatic syndromes include motility disorders of collagenosis, mixed connective tissue disease and Sjögrens syndrome associated with the risk of esophagitis due to gastroesophageal reflux. This is in addition to the nonsteroidal anti-inflammatory drug-induced gastroduodenal lesions in childhood and adolescence. However, the relevance of co-medication with steroids for the pathogenesis of these lesions is controversial [8].

As for pediatric rheumatologic emergencies, Henoch-Schönlein purpura, polyarteritis nodosa, Wegener granulomatosis, systemic lupus erythematosus and juvenile idiopathic arthritis are generally associated with abdominal complications. Again, abdominal pain is a very common complaint of children in the emergency department. Abdominal pain triggered by rheumatologic disorders mainly stems from the gastrointestinal system and the abdominal vasculature. Bowel ischemia or edema is the leading cause of acute abdomen in children diagnosed with vasculitis. The small intestine is the most commonly affected part of the gastrointestinal system, followed by the mesentery and colon. Furthermore, gastrointestinal system hemorrhage, bowel perforation and bowel infarction are not rare presentations [9].

Gastro-hepatic manifestations in RDs are not rare, so clinicians should be aware of their existence and 
the fact that they may occur concomitantly or serially. Furthermore, understanding the range and prevalence of GI manifestations associated with RDs, with related autoimmune disorders and with RDs treatments is essential for rheumatologists and other clinicians caring for patients with RDs And that is why the current study was suggested ${ }^{[3] \text {. }}$

This study aimed to discuss and summarize current data on gastro-intestinal manifestations encountered by rheumatologic diseases in pediatric patients.

\section{Gastrointestinal Tract Role in the Pathogenesis of Rheumatic Diseases}

Autoimmune rheumatic diseases (ARDs) encompass a wide variety of illnesses in which innate and adaptive immune responses lead to autoimmunemediated tissue damage. In pediatrics, Juvenile rheumatoid arthritis (JRA) is a generic term for arthritis that has an onset before the age of 16 and persists for more than 6 weeks. The JRA nomenclature represents an exclusion diagnosis that includes all forms of chronic childhood arthritis of unknown origin. JRA is the most common chronic rheumatic illness in children and is a significant cause of both short- and long-term disabilities. The heterogeneity of this disease suggests that different factors likely contribute to its pathogenesis. The current understanding of JRA indicates that it arises in a genetically susceptible individual due to environmental factors. Moreover, it has been proposed that an antigen-driven autoimmune process mediates the inflammatory pathology of some cases of arthritis (e.g., oligoarthritis, polyarthritis). In contrast, there are no signs of lymphocyte-mediated, antigen-specific immune responses in individuals with systemic onset disease $[10,11]$.

\section{Digestive System Complications from Rheumatic Diseases}

In children, rheumatic diseases are mostly chronic inflammatory diseases with an autoimmunological pathophysiology involving different tissues and organs. Manifestations of this disease, including arthritis, synovitis, conjunctivitis, iridocyclitis, vasculitis, myositis, dermatitis, or mucositis can be observed in many organs. The primary gastrointestinal manifestation of a rheumatic disease seems caused by an inflammation of the gastrointestinal mucosa, respectively its small vessels, the connective tissue or the muscles of the intestinal wall. These processes of inflammation can destroy the normal structures of the mucosa and/or the gastrointestinal muscularis causing impaired function followed by symptoms of malabsorption, maldigestion or gastrointestinal motility disorders. In addition, drugs used for antiinflammatory treatment such as non-steroidal antiinflammatory drugs (NSAID), steroids or methrotrexat are characterized by a spectrum of adverse gastrointestinal side effects, including the affection of the mucosa $[12,13]$.

\section{Gastrointestinal Symptoms of Children with Rheumatic Diseases}

Although several reports have established that gastrointestinal symptoms are frequent in adults with inflammatory rheumatic diseases, systematic studies to investigate the prevalence of gastrointestinal symptoms and gastrointestinal involvement in children and adolescents with rheumatic diseases are rare $^{(14)}$.

1. Abdominal pain is the most frequent symptom reported. However, little data exists about gastrointestinal symptoms which arise in children with rheumatic diseases not treated with a medication (such as non-steroidal antiinflammatory drugs) that could also induce abdominal pain. Dowd et al. [15] investigated the prevalence of abdominal pain in 570 children with a mean age of 9 years and a dominant diagnosis of oligoarticular and polyarticular arthritis. Of the children not taking non-steroidal drugs, $15 \%$ reported abdominal pain. This data was collected retrospectively from patient charts. Assuming that outpatient children with rheumatic disease and their parents report gastrointestinal complaints only if they are severe or if explicitly asked, this data could underestimate the real prevalence of pain. Weber et al. [12] prospectively investigated 41 children and adolescents with a mean age of 12.3 years (ranging from 8.0 to 18.5 years) who had a systemic form of juvenile idiopathic arthritis, persistent oligoarthritis, polyarthritis (rheumatoid factor negative), or enthesitis-related arthritis. 27 of the 41 children $(>50 \%)$ reported chronic abdominal pain, $30 \%$ reported pain once a month, $27 \%$ reported pain at least once a week. In eight patients, the pain was so strong that daily activities were stopped, 12 missed school due to abdominal pain, and 8 took analgetic drugs. In contrast, dyspepsia, vomiting, loss of appetite, meteorism, regurgitation, retrosternal pain, or diarrhea are rarely reported complaints. In this study, however, all patients took a non-steroidal anti-inflammatory drug. Therefore, these findings show the relevance of gastrointestinal symptoms in children with a chronic arthritis under treatment, but cannot confirm the hypothesis of a primary gastrointestinal inflammation [16].

2. Oral ulcers: Rheumatological Causes: [17].
A. Rheumatological diseases
B. Drug induced
C. Dysphagia: Causes (17)

\section{Gastrointestinal Inflammation in Pediatric} Rheumatic Diseases

Patients with a connective tissue disease or vasculitis are at risk of gastrointestinal complications (18).

\section{Spondyloarthropathies (SpA):}

SpA include many different forms of inflammatory arthritis and can affect the spine (axial 
SpA) and/or peripheral joints (peripheral SpA) with Ankylosing spondylitis (AS) being the prototype of the former. Extra-articular manifestations, like uveitis, psoriasis and inflammatory bowel disease (IBD) are frequently observed in the setting of $\mathrm{SpA}$ and are, in fact, part of the SpA classification criteria. Bowel involvement seems to be the most common of these manifestations. Clinically evident IBD is observed in $6 \%-14 \%$ of AS patients, which is significantly more frequent compared to the general population. Besides, it seems that silent microscopic gut inflammation, is evident in around $60 \%$ in AS patients. Interestingly, occurrence of IBD has been associated with AS disease activity. For peripheral SpA, two different forms have been proposed with diverse characteristics. Of note, SpA (axial or peripheral) is more commonly observed in Crohn's disease than in ulcerative colitis. The common pathogenetic mechanisms that explain the link between IBD and SpA are still ill-defined [19, 20].

\section{Jeuvenile Systemic lupus erythematosus (jSLE):}

Juvenile-onset systemic lupus erythematosus (jSLE) accounts for up to $20 \%$ of all SLE patients. Key differences between juvenile- and adult-onset (aSLE) diseases include higher disease activity, earlier development of damage, and increased use of immunosuppressive treatment. Regarding children with systemic lupus erythematosus: up to $15 \%$ show an oral or nasopharyngeal ulceration, up to $10 \%$ a mesenteric thrombosis, and up to $5 \%$ a sterile peritonitis.Up to $50 \%$ of patients with systemic lupus suffer from gastrointestinal symptoms like nausea, vomiting, dysphagia, diarrhea or abdominal pain. The whole gastrointestinal tract could be involved in the inflammatory process; however with lupus enteritis most reported manifestations are located in the ileum or jejeunum [21, 22].

\section{Churg-Strauss syndrome:}

The Churg-Strauss syndrome occurs very rarely in childhood. This disease is an allergic granulomatousis angiitis followed by a necrotizing vasculitis. Gastrointestinal symptoms like abdominal pain or diarrhea are found in up to $44 \%$ of patients. Eosinophilic infiltration of the intestinal mucosa has been reported, while ulcerations and perforation are very rare [23]. A predominant involvement of the colon is discussed. Henoch-Schönlein purpura is a systemic small vessel vasculitis involving the skin, some joints, the kidneys, and the gastro-intestinal tract. This vasculitis is an IgA-mediated vasculitis and is characterized by IgA deposits in the small vessels of the gastrointestinal tract [24]

Behçet syndrome:

It is characterized by multiorganic and chronic, recurrent inflammation mainly manifested in oral aphthous ulcers, genital ulcerations and uveitis. Gastrointestinal (GI) system involvement in children with BD varies between 4.8 and $56.5 \%$ [25]. It has been reported that GI involvement is more common in children than in adults [26]. Gastrointestinal symptoms usually start within 4.5-6 years after the onset of oral ulcers [27]. Although mucosal lesions may occur in any part of the digestive track, the ileocecal region is most frequently involved. The most common symptoms are abdominal pain, nausea, vomiting, dyspepsia, diarrhea, and gastrointestinal bleeding [28]. It is difficult to differentiate the GI involvement of BD from inflammatory bowel diseases. The round ulcers, the focal single / multiple distribution patterns, $<6$ ulcers, and the absence of a cobblestone appearance were found to be related with BD. Intestinal ischemia due to arterial involvement and Budd-Chiari syndrome associated with venous involvement are other gastrointestinal manifestations [27].

\section{Kawasaki syndrome:}

It is an acute systemic inflammatory multi-organ disease with a vasculitis of medium-sized and small vessels. The involvement of the coronary arteries is critical. The etiology of Kawasaki disease is unknown. Diagnosis is based on the following symptoms (diagnosis occurs when 5 of the 6 symptoms are present): a) persistent fever lasting over 5 days, b) bilateral nonpurulent conjunctivitis, c) polymorphous rash, d) nonsuppurative cervical lymphadenopathy, e) erythema and/or, oedema and/or desquamation of the hand and feet, f) injected or fissured lips and/or injected pharynx and/or strawberry tongue. These symptoms cannot be explained by any other known disease [29]. Gastrointestinal symptoms are not unusual features, particularly stomatitis, paralytic ileus, and hydrops of the gallbladder. Up to $5 \%$ of children with Kawasaki syndrome have an acute abdomen. Abdominal pain, distension, vomiting, hepatomegaly, and jaundice are the most common features at onset in the subgroup of children with Kawasaki syndrome needing surgical intervention [30]. In addition, inflammation may cause pseudoobstruction, which leads to mechanical ileus [29].

\section{Sjörgen's syndrome (SS):}

The gastrointestinal manifestation of Sjörgen's syndrome (SS) includes difficulties in chewing, initial swallowing, and an increased frequency of dental caries. Furthermore, dysphagia due to the lack of saliva as well as esophageal dysmotility and chronic atrophic gastritis probably account for epigastric pain, nausea, and other dyspeptic symptoms [31]. The degree to which SS affects the small and large bowel is unclear. In the gastrointestinal dysfunction of the $\mathrm{SS}$, abnormalities of the autonomic nervous system seem to be involved. As in systemic sclerosis, antibodies reacting with the $\mathrm{m} 3$ subtype muscarinic acetylcholine receptor appear to play an important role [30].

5. Gastrointestinal Motility Disorders in Pediatric Rheumatic Diseases

Symptoms:

The motility dysfunction can involve the whole gastrointestinal tract from esophagus to rectum. Gastroesophageal reflux, gastroparesis, pseudo- 
obstruction, bacterial overgrowth, diverticula and anorectal dysmotility has been described in adults. $43 \%$ of patients with juvenile-onset mixed connective tissue disease suffer from dysphagia. Heartburn is the second most reported symptom of esophageal involvement. Esophageal dysmotility is the most common internal organ manifestation in children with systemic scleroderma [32]. Abnormalities included lower esophageal sphincter pressure as well as tertiary pressure waves, contraction waves with low amplitudes, and simultaneous, non-propulsive contraction in different parts of the esophagus [33]. Using the 24-hour pH-metry, $64 \%$ of patients showed an elevated reflux index, $85 \%$ an increased number of refluxes and $50 \%$ an increased number of refluxes longer than 5 minutes, which is the most important factor for predicting developing esophagitis. Such abnormalities were observed in children with mixed connective tissue disease and systemic scleroderma as well as in children with localized sclerderma [34].

Causes:

Gastrointestinal motility abnormalities are well recognized in scleroderma, mixed connective tissue disease, dermatomyositis, and systemic lupus erythematosus [35].

6. Gastrointestinal Side Effects of Non-steroidal Anti-inflammatory Drugs in Children

As it has been shown in adults, nonsteroidal antiinflammatory drugs (NSAID) cause gastroduodenal injuries such as gastritis, ulceration in the upper and lower gastrointestinal tract, and hemorrhage in a large number of patients. Although the incidence of NSAID-related severe gastrointestinal complications seems to be less frequent during the last decade, 10 to 20 percent of patients have dyspepsia while taking NSAID. Although increased risk of gastrointestinal toxicity of NSAID is associated with advanced age, beside some case reports. several studies were performed to investigate the prevalence of gastrointestinal lesions in children taking NSAID [36]. In a network meta-analysis to compare the efficacy and safety of non-steroidal anti-inflammatory drugs (NSAIDs) in treating patients with juvenile idiopathic arthritis, the most common adverse effects across all treatment groups were gastrointestinal side effects, rash, headache, and pyrexia. These side effects occurred more frequently within the aspirin, tolmetin, and ibuprofen groups, resulting in more noncompliance. Estimates of NSAIDs-associated gastropathy range from $0.7-75 \%$, depending on different study designs. Most of the gastrointestinal disorders were mild, while serious gastropathy such as gastrointestinal perforation and massive gastrointestinal hemorrhage was lower than adults. The combination of glucocorticoid, leflunomide, and methotrexate can aggravate gastrointestinal adverse reactions. While children have a very low risk of cardiovascular thromboembolic and serious gastrointestinal events, prolonged use of NSAIDS into adulthood could make them vulnerable to such risks, especially when associated with other risk factors such as obesity or smoking [37]. In medical charts of 702 children with juvenile rheumatic arthritis treated in a department of pediatric rheumatology over a period of 15 years. Only 5 children developed a clinically significant gastropathy. In each child two episodes were documented, 7 episodes were associated with an intake of tolmetin, two episodes with aspirin and one episode with diclofenac. The clinical symptoms were caused by esophagitis or duodenal and gastric ulcers. All children responded to an antiulcer treatment and were symptom-free after discontinuing NSAID treatment [38].

NSAID treatment seems to be associated with a significant increase of gastroduodenal lesions in children with juvenile idiopathic arthritis. Abdominal pain was the leading gastrointestinal symptom of these adverse effects. In the small number of endoscopically and histologically studies done, abdominal pain seems to be associated with the presence of gastroduodenal lesions. Neither co-medication with steroids nor Helicobacter pylori infection seems to be additional risk factors for gastroduodenal lesions. In addition, no sufficient data exists regarding the effectiveness of a drug to treat or prevent NSAID-induced lesions in children. COX-2 inhibitors reported a lower incidence of gastrointestinal lesions but a higher risk of severe cardiovascular side effects (39). Further prospective studies using invasive and noninvasive procedures are needed. Furthermore, the psychosomatic aspect of recurrent abdominal pain in children with a chronic disease should be taken into account ${ }^{[40] \text {. }}$

\section{Hepatic Manifestations of PEDIATRIC Rheumatic Diseases}

The autoimmune connective tissue diseases have a complex pathogenesis with a multifactorial etiology. In the course of these diseases develop an autoimmune response leading to chronic inflammation and that sometimes may cause multiorgan dysfunction. The liver is a life-sustaining organ which is responsible for detoxification of drugs and other harmful substances, metabolism of hormones, storage and release of proteins, cholesterol and vitamins and also an active organ of immune response [19].

Liver injury such as hepatomegaly, splenomegaly and various degrees of biochemical abnormalities are quite common in children with collagen vascular diseases. They may be primary (vascular infiltration or thrombosis) or secondary, particularly due to drug therapy (drug toxicity, fatty infiltration), superadded infections, diabetes or overlap with autoimmune hepatitis [41].

\section{Causes}

\section{Systemic lupus erythematosus}

Juvenile-onset systemic lupus erythematosus (jSLE) accounts for up to $20 \%$ of all SLE patients. Key differences between juvenile- and adult-onset disease include higher disease activity, earlier development of damage, and increased use of 
immunosuppressive treatment in jSLE suggesting infectivity secondary to variable pathomechanisms [42].

\section{Anti-phospholipid syndrome}

Antiphospholipid syndrome (APS) (Hughes syndrome) is an autoimmune disease marked by arterial or venous thrombosis, thrombocytopenia and presence of antiphospholipid antibodies (APL) (such as lupus anticoagulant, anticardiolipin or b2glycoprotein) [43].

\section{Juvenile idiopathic arthritis}

(JIA), Juvenile idiopathic arthritis (JIA), is the most common, chronic rheumatic disease of childhood, affecting approximately one per 1,000 children. Disease onset is before 16 years of age [44].

Macrophage activation syndrome (MAS):

MAS is a severe complication of rheumatic disease in childhood, particularly in systemic Juvenile Idiopathic Arthritis (sJIA). It is characterize by an uncontrolled activation and proliferation of $\mathrm{T}$ lymphocytes and macrophages. MAS identifies a potentially fatal complication of rheumatic diseases. It occurs usually in the context of systemic Juvenile Idiopathic Arthritis (sJIA), but it may occur also, albeit more rarely, in systemic Lupus Erythematosus and Kawasaki disease [45].

\section{Felty's syndrome (FS)}

It is rarely seen in patients with juvenile idiopathic arthritis (JIA)(46). Nodular regenerative hyperplasia of the liver can cause portal hypertension and in consequence esophageal variceal bleeding [47]. The splenectomy may be beneficial in the treatment of portal hypertension [48].

\section{Primary Sjögren's syndrome(pSS)}

Sogrens Syndrome (SS) is an uncommon condition in the pediatric age group. SS is a chronic autoimmune disorder mainly affects salivary and lacrimal glands, with varying degrees of systemic involvement. SS occurs usually after 40 years and nine times more common in females [49].

\section{Juvenile-onset systemic sclerosis (jSSc)}

Juvenile-onset systemic sclerosis (jSSc) is a rare and severe autoimmune disease with associated lifethreatening organ inflammation and evidence of fibrosis. The organ manifestations of jSSc resemble adult SSc, but with better outcomes and survival. Systemic sclerosis (SSc) is a chronic systemic connective tissue disease which presents with progressive fibrosis of skin and internal organs and also injuries of small arteries (vasculopathy) [50].

\section{The juvenile idiopathic inflammatory myopathies (JIIMs)}

The idiopathic inflammatory myopathies are a heterogenic group of muscle diseases with diverse symptoms and etiology [51]. The juvenile idiopathic inflammatory myopathies (JIIMs) are heterogeneous, systemic autoimmune diseases characterized by weakness, chronic inflammation of skeletal muscles, and typical skin rashes (Gottron's papules or heliotrope rash) with onset during childhood. The criteria established by Bohan and Peter based on these features [52], as well as the presence of elevated serum levels of muscle enzymes or increased electrical activity in the muscle detected by electromyography, have been used to diagnose these disorders. However, new classification criteria have recently been developed and validated [51]. Evidence from several large JIIM registry studies has led to increased understanding of the spectrum of phenotypes associated with JIIMs, based on either clinicopathologic features or the presence of autoantibodies found almost exclusively in patients with myositis (known as myositis autoantibodies) [53, 54].

\section{Polyarteritis nodosa (PAN):}

Childhood-onset polyarteritis nodosa (PAN) is a rare and systemic necrotising vasculitis in children affecting small- to medium-sized arteries that causes organ damage in the gastrointestinal system in more than $50 \%$ of cases [55]. The liver can also be affected in the course of PAN and it can manifest as hepatomegaly, jaundice, cholestasis without jaundice and even as massive hepatic necrosis. There are two subsets of PAN: primary of unknown etiology and secondary associated with presence of hepatitis B virus (HBV-related PAN)[56]

\section{Hepatotoxicity of drugs used in rheumatology}

Drug-induced liver injury (DILI) is the main reason for drugs' withdrawal from clinical trials or even from market registered substances. It may occur as a direct result of a drug or its metabolites toxicity affecting hepatocytes (intrinsic DILI) or through immune activation (idiosyncratic DILI). Intrinsic DILI is predictable (occurs in a large proportion of exposed individuals), dose related and occurs within hours or days [19].

\section{Pancreatic Manifestations of Pediatric Rheumatic Diseases}

Acute pancreatitis (AP) is an acute inflammatory process of the pancreas characterized by significant abdominal pain, nausea and vomiting [57]. Chronic pancreatitis (CP) is characterized by chronic inflammation and fibrosis of the pancreas. Autoimmune pancreatitis (AIP) is a distinct category and has been associated with systemic, autoimmune inflammatory disorders; with type 1 AIP presenting as a syndrome involving several organs, and type 2 is associated with inflammatory bowel disease. Pancreatic cancer $(\mathrm{PaC})$ is the second most common gastrointestinal (GI) malignancy. While the aetiology remains unclear, chronic inflammation has emerged as a key mediator of $\mathrm{PaC}$ development. Several case reports and studies have described an association between Sjo“ gren's syndrome (both primary and secondary) and RA with pancreatitis patients [58].

Pancreatic Manifestations of RA:

Patients with RA had more severe features of AP including, ileus, abdominal compartment syndrome, sepsis, DIC and shock. They also had a higher risk of complications at 30 days including infections, 
pseudocyst, pulmonary embolism and acute coronary syndrome. Similarly, patients with RA who developed CP had worse outcomes compared with the general population, including more psychiatric conditions, pain medication requirement, bone disease and exocrine dysfunction [59].

\section{Pancreatic Manifestations of SLE:}

Acute pancreatitis is a rare but dreaded complication of SLE. It can have varied presentations. It may be an initial presentation of SLE or can present as its flare. It must be suspected in any patient of SLE presenting with acute abdominal pain. Conversely, in any young female presenting with acute pancreatitis, SLE must be considered. Because development of acute pancreatitis is a function of disease activity of SLE, it is imperative that a tight control of its activity will go a long way in preventing this dangerous complication. Finally, in SLE with acute pancreatitis, APS and AIHA should be considered as predisposing factors, be investigated, and treated appropriately. Rituximab seems to be a promising drug for this association. A thought can also be given for prevention of acute pancreatitis, especially if SLE is associated with AIHA or APS. This can be achieved by strict control of the disease activity, and may be, by using rituximab [60, 61].

\section{Pancreatic Manifestations of Sjogren's Syndrome Acute pancraetitius:}

$\mathrm{SS}$ is an autoimmune disease involving exocrine glands. Chronic pancreatitis has been found in association with other autoimmune diseases such as SS, primary biliary cirrhosis, and sclerosing cholangitis. A study on the association between PSS and acute pancreatitis showed that PSS may increase the acute pancreatitis risk, but the study population excluded individuals with coexisting autoimmune disease [62].

\section{Chronic pancreatitis}

Pancreatitis was documented in $7 \%$ of patients with SS. It might present as autoimmune pancreatitis or chronic pancreatitis. There are multiple reported cases of SS with pancreatic calcifications. Enlarged pancreatic head suggestive of neoplasm and increased serum CA 19-9 antibodies in benign pancreatic processes had been also reported. Pancreatic exocrine insufficiency is not uncommon, and it is related to reduced gastric secretions and/or abnormal gallbladder function [63].

Primary sclerosing cholangitis (PSC) and secondary sclerosing cholangitis :

They are associated with chronic pancreatitis and SS or sicca syndrome. Treatment depends on sclerosing cholangitis status and the degree of extrahepatic involvement. Immunomodulators (including steroids, azathioprine, and rituximab) are the mainstay of treatment for autoimmune sclerosing cholangitis with or without autoimmune pancreatitis. Endoscopic treatment is directed to therapeutic intervention to release the biliary obstruction and for tissue sampling. Liver transplantation is the treatment for end-stage liver disease due to sclerosing cholangitis or recurrent cholangitis [64, 65, and 66].

\section{Conclusion}

The importance of the gastrointestinal (GI) tract in development of autoimmunity has been increasingly appreciated in rheumatological diseases. The small intestine is the most commonly affected part of the gastrointestinal system. Abnormalities in liver function tests are very common in patients with rheumatic diseases and should be further evaluated.

\section{References}

[1] Kröner PT, Tolaymat OA, Bowman AW, Abril A, Lacy BE. Gastrointestinal Manifestations of Rheumatological Diseases. Am J Gastroenterol. 2019 Sep;114(9):1441-1454.

[2] Ohira, Hiromasa; Migita, Kiyoshi (2019). Gastrointestinal and Hepatic Manifestations of Rheumatic Diseases || . , 10.1007/978-981-136524-9(),-

[3] E.Craig, L.C.Cappelli, Gastrointestinal and Hepatic Disease in Rheumatoid Arthritis. Rheumatic Disease Clinics.vol.44(1),pp. 89$111,2018$.

[4] F.Ciccia, A.Accardo-Palumbo, A.Rizzo, G.Guggino, S.Raimondo, A.Giardina, A. Cannizzaro, RA.Colbert, R.Alessandro, G.Triolo. Evidence that autophagy, but not the unfolded protein response, regulates the expression of IL-23 in the gut of patients with ankylosing spondylitis and subclinical gut inflammation. Ann Rheum Dis.vol.73,pp.1566$1574,2014$.

[5] Ross CB, Scott HW, Pincus T. Jejunoileal bypass arthritis. Baillieres Clin Rheumatol. 1989 Aug;3(2):339-55.

[6] V.Moos, T.Schneider. Changing paradigms in Whipple's disease and infection with Tropheryma whipplei. European journal of clinical microbiology \& infectious diseases.vol. 30(10),pp. 1151-1158,2011.

[7] S. K.Schmitt, Reactive arthritis. Infectious Disease Clinics.vol.31(2),pp.265-277,2017.

[8] P. Weber, T.Brune, G.Ganser, K.Zimmer. Gastrointestinal symptoms and permeability in patients with juvenile idiopathic arthritis. Clinical and experimental rheumatology.vol. 21(5), pp.657-662,2003.

[9] S.Ozen, A.Pistorio, SM.Iusan, Bakkaloglu A, Herlin T, Brik R, et al. EULAR/PRINTO/PRES criteria for Henoch-Schönlein purpura, childhood polyarteritis nodosa, childhood Wegener granulomatosis and childhood Takayasu arteritis: Ankara 2008. Part II: Final classification criteria. Ann Rheum Dis. May.vol.69(5),pp.798806,2010.

[10] d'Angelo, G.Di Donato, L.Breda, F.Chiarelli, Growth and puberty in children with juvenile 
idiopathic arthritis. Pediatric Rheumatology.vol.19(1),pp.28,2021.

[11] Bank I. The Role of Gamma Delta T Cells in Autoimmune Rheumatic Diseases. Cells. 2020;9(2):462. Published 2020 Feb 18.

[12] W.Peter, D.Bolz, Z.Klaus-Peter, Gastrointestinal Involvement in Children and Adolescents with Rheumatic Diseases. Current Rheumatology Reviews.vol.2(2),pp.207213,2006.

[13] Pohl D, Benseler S. Systemic inflammatory and autoimmune disorders. Handb Clin Neurol. 2013;112:1243-52. D.M.

[14] Chong VH, Wang CL. Higher prevalence of gastrointestinal symptoms among patients with rheumatic disorders. Singapore Med J. 2008 May;49(5):419-24. PMID: 18465055.

[15] J.E.Dowd, R.Cimaz, C.W.Fink, Nonsteroidal antiinflammatory drug-induced gastroduodenal injury in children. Arthritis \& Rheumatism: Official Journal of the American College of Rheumatology.vol.38(9),pp.1225-1231,1995.

[16] Silverstein FE, Faich G, Goldstein JL, Simon LS, Pincus T, Whelton A, Makuch R, Eisen G, Agrawal NM, Stenson WF, Burr AM, Zhao WW, Kent JD, Lefkowith JB, Verburg KM, Geis GS. Gastrointestinal toxicity with celecoxib vs nonsteroidal anti-inflammatory drugs for osteoarthritis and rheumatoid arthritis: the CLASS study: A randomized controlled trial. Celecoxib Long-term Arthritis Safety Study. JAMA. 2000 Sep 13;284(10):1247-55.

[17] H.Halabi, A.AlDabbagh, A.Alamoudi, Gastrointestinal Manifestations of Rheumatic Diseases Skills in Rheumatology Springer,Singapore,pp.475-499,2021.

[18] Shimoda, Shinji \& Chong, Yong \& Akahoshi, Mitsuteru \& Niiro, Hiroaki \& Tsukamoto, Hiroshi. (2016). Hepatic and Gastrointestinal Manifestations in Rheumatic and Connective Tissue Diseases. Journal of General and Family Medicine. 17. 132-137.

[19] J.Podgórska, P.Werel, J.Klapaczyński, D.Orzechowska, M.Wudarski, A.Gietka, Liver involvement in rheumatic diseases. Reumatologia.vol.58(5),pp.289-296,2020.

[20] G.E.Fragoulis, C.Liava, D.Daoussis, E.Akriviadis, A.Garyfallos, T.Dimitroulas, Inflammatory bowel diseases and spondyloarthropathies: From pathogenesis to treatment. World J Gastroenterol.vol.25(18),pp.2162-2176,2019.

[21] Poddubnyy D. Axial spondyloarthritis: is there a treatment of choice?. Ther Adv Musculoskelet Dis. 2013;5(1):45-54

[22] C.Lee, M.Ahn, E.Y.Lee, J.Shin, Y.Cho, H.Ha, B.Yoo, H.Moon, Acute abdominal pain in systemic lupus erythematosus: focus on lupus enteritis (gastrointestinal vasculitis). Annals of the rheumatic diseases.vol.61(6),pp.547$550,2002$.

[23] Smith EMD, Lythgoe H, Midgley A, Beresford MW, Hedrich CM. Juvenile-onset systemic lupus erythematosus: Update on clinical presentation, pathophysiology and treatment options. Clin Immunol. 2019 Dec;209:108274

[24] Y.Ito, M.Yoshida, T.Sugiyama, H.Masuda, M.Mori, N.Kimura, M.Umakoshi, K.Miyabe, Y.Kudo-Asabe, A.Goto, Multiple ulcerations and perforation in the small intestine after steroid treatment in eosinophilic granulomatosis with polyangiitis: a case report and literature review. Cardiovascular Pathology.vol.47,pp.107193,2020.

[25] A.Kopparapu, D.Jarrett, S.Kraleti, IgA vasculitis presenting as abdominal pain and rash. Proc (Bayl Univ Med Cent).vol.32(4),pp.599600,2019.

[26] Y.-C.Hu, B.-L.Chiang, Y.-H.Yang, Clinical Manifestations and Management of Pediatric Behçet's Disease. Clinical Reviews in Allergy \& Immunology,pp.1-10,2020.

[27] E.Seyahi, H.Özdoğan, Juvenile Behçet's syndrome. In Y. H. Yazici Y (Ed.), Behçet's syndrome New York, NY: Springer,pp.205$214,2010$.

[28] W.Skef, M.J.Hamilton, T.Arayssi, Gastrointestinal Behçet's disease: a review. World Journal of Gastroenterology.WJG.vol.21(13),pp.3801,2015.

[29] D.Mendes, M.Correia, M.Barbedo, T.Vaio, M.Mota, O.Gonçalves, J.Valente, Behçet's disease-a contemporary review. Journal of autoimmunity.vol.32(3-4),pp.178-188,2009.

[30] D.Bagrul, E.G.Karadeniz, S.Koca, Gastrointestinal involvement in Kawasaki disease: a case report. Cardiology in the Young.vol.28(8),pp.1070-1073,2018.

[31] P.Nasri, Z.Adibmajlesi, H.Rahimi, H.Saneian, F.Famouri, M.Khademian, M.Jafarinia, F.Alsahebfosoul, Gastrointestinal Manifestations in Children with Kawasaki Disease in Isfahan, Iran. Archives of Pediatric Infectious Diseases.vol.8(2),2020.

[32] S.Parreau, J.Jacques, S.Dumonteil, S.Palat, S.Geyl, G.Gondran, H.Bezanahary, E.Liozon, J.Azaïs, S.Colombie, M.-O.Jauberteau, V.Loustaud-Ratti, K.-H.Ly, A.-L.Fauchais, Abdominal symptoms during Sjogren's syndrome: a pilot study. Advances in Rheumatology.vol.61(1),pp.5,2021.

[33] K.Denaxas, S.D.Ladas, G.P.Karamanolis, Evaluation and management of esophageal manifestations in systemic sclerosis. Ann Gastroenterol.vol.31(2),pp.165-170,2018.

[34] N.J.Sheehan, Dysphagia and other manifestations of oesophageal involvement in the musculoskeletal diseases. Rheumatology.vol.47(6),pp.746-752,2008. 
[35] J.Raja, C.T.Ng, I.Sujau, K.F.Chin, S.Sockalingam, High-resolution oesophageal manometry and 24-hour impedance-pH study in systemic sclerosis patients: association with clinical features, symptoms and severity. Clinical and experimental rheumatology.vol.34(5),pp.115-121,2016.

[36] T.-Y.Chi, H.-M.Zhu, M.Zhang, Risk factors associated with nonsteroidal anti-inflammatory drugs (NSAIDs)-induced gastrointestinal bleeding resulting on people over 60 years old in Beijing. Medicine.vol.97(18),pp.e0665,2018.

[37] C.-1.Shi, Y.Zhang, Z.-y.Zhang, J.Zhou, X.M.Tang, Comparative Efficacy and Safety of Non-Steroidal Anti-Inflammatory Drugs in Patients With Juvenile Idiopathic Arthritis: A Systematic Review and Network Meta-analysis. Indian Pediatrics.vol.58(2),pp.162-168,2021.

[38] G.Keenan, E.Giannini, B.Athreya, Clinically significant gastropathy associated with nonsteroidal antiinflammatory drug use in children with juvenile rheumatoid arthritis. The Journal of rheumatology.vol. 22(6),pp.11491151,1995 .

[39] J.S.Borer, L.S.Simon, Cardiovascular and gastrointestinal effects of COX-2 inhibitors and NSAIDs: achieving a balance. Arthritis Res Ther.vol.7,pp.S14-S22,2005.

[40] X.Ioannis, M.Antigoni, N.Natalia, V.Konstantina, K.Ioanna, The role of psychosocial factors in children with recurrent abdominal pain. Pediat Therapeut.vol.3(170),pp.2161-0665,2013.

[41] J.Pawłowska, M.Naorniakowska, A.Liber, Liver involvement in children with collagen vascular diseases. Clin Exp Hepatol.vol.1(3),pp.117-119,2015.

[42] E.M.D.Smith, H.Lythgoe, A.Midgley, M.W.Beresford, C.M.Hedrich, Juvenile-onset systemic lupus erythematosus: Update on clinical presentation, pathophysiology and treatment options.

Clinical Immunology.vol.209,pp.108274,2019.

[43] S.Miyakis, M.Lockshin, T.Atsumi, D.Branch, R.Brey, R.Cervera, R.Derksen, P.De Groot, T.Koike, P.Meroni, International consensus statement on an update of the classification criteria for definite antiphospholipid syndrome (APS). Journal of thrombosis and haemostasis.vol.4(2),pp.295-306,2006.

[44] manners PJ, Diepeveen DA. Prevalence of juvenile chronic arthritis in a population of 12-year-old children in urban Australia. Pediatrics 1996;98:84-90.

[45] C.Bracaglia, G.Prencipe, F.De Benedetti, Macrophage Activation Syndrome: different mechanisms leading to a one clinical syndrome. Pediatr Rheumatol Online J.vol.15(1),pp.55,2017 .
[46] G.-m.Li, H.-m.Liu, W.-z.Guan, Y.-f.Li, H.Xu, L.Sun, Phenotypic variability in a child with Felty's syndrome: a case report. BMC Pediatrics.vol.20,2020.

[47] J.Bosch, Salvage transjugular intrahepatic portosystemic shunt: is it really life-saving? Journal of hepatology.vol.35(5),pp.658$660,2001$.

[48] H.Stock, Z.Kadry, J. P.Smith, Surgical management of portal hypertension in Felty's syndrome: a case report and literature review. Journal of hepatology.vol.50(4),pp.831$835,2009$.

[49] K.J.Kumar, A.S. Kudakesseril, C.S.Sheeladevi, H.V.Sowmya, Primary Sjogrens Syndrome in a Child. Iranian journal of pediatrics.vol.25(2),pp.e254-e254,2015.

[50] A.M.Stevens, K.S.Torok, S.C.Li, S.F.Taber, T.T.Lu, F.Zulian, Immunopathogenesis of Juvenile Systemic Sclerosis. Front Immunol.vol.10,pp.1352-1352,2019.

[51] I.E.Lundberg, F.W.Miller, A.Tjärnlund, M.Bottai, Diagnosis and classification of idiopathic inflammatory myopathies. J Intern Med.vol.280(1),pp.39-51,2016.

[52] A.Bohan, J.B.Peter, Polymyositis and dermatomyositis (first of two parts). The New England journal of medicine.vol.292(7),pp.344347,1975 .

[53] A.Tjärnlund, M.Bottai, L.Rider, V.Werth, C.Pilkington, M.de Visser, L.Alfredsson, A.Amato, R.Barohn, M.Liang, Progress Report On Development of Classification Criteria for Adult and Juvenile Idiopathic Inflammatory Myopathies.: $753 . \quad$ Arthritis \& Rheumatism.vol.64,2012.

[54] L.G.Rider, K.Dankó, F.W.Miller, Myositis registries and biorepositories: powerful tools to advance clinical, epidemiologic and pathogenic research. Current opinion in rheumatology.vol.26(6),pp.724-741,2014.

[55] L.G.Rider, J.D.Katz, O.Y.Jones, Developments in the classification and treatment of the juvenile idiopathic inflammatory myopathies. Rheumatic diseases clinics of North America.vol.39(4),pp.877-904,2013.

[56] J.-S.Lee, J.-G.Kim, S.Lee, Clinical presentations and long term prognosis of childhood onset polyarteritis nodosa in single centre of Korea. Scientific reports.vol.11(1),pp.8393,2021.

[57] E.C.Ebert, K.D.Hagspiel, M.Nagar, N.Schlesinger, Gastrointestinal involvement in polyarteritis nodosa. Clinical Gastroenterology and Hepatology.vol.6(9),pp.960-966,2008.

[58] A.Garber, C.Frakes, Z.Arora, P.Chahal, Mechanisms and management of acute pancreatitis. Gastroenterology research and practice, 2018. 
[59] C.-C.Chang, Y.-S.Chang, S.-H.Wang, S.Y.Lin, Y.-H.Chen, J.H.Chen, Primary Sjogren's syndrome and the risk of acute pancreatitis: a nationwide cohort study. BMJ open.vol.7(8),pp.e014807,2017.

[60] M.Alkhayyat, M.Abou Saleh, M.K.Grewal, M.Abureesh, E.Mansoor, C.R.Simons-Linares, A.Abelson, P.Chahal, Pancreatic manifestations in rheumatoid arthritis: a national populationbased study. Rheumatology.vol.60(5),pp.2366$2374,2021$.

[61] Jia Y, Ortiz A, Mccallum R, Salameh H, Serrato P. Acute pancreatitis as the initial presentation of systematic lupus erythematosus. Case Rep Gastrointest Med. 2014;2014:571493

[62] P.Jadhav, S.Desai, J.Jadhav, L.Bichile, Acute pancreatitis in rheumatology practice, with emphasis on systemic lupus erythematosus: A case series and newer concepts. Indian Journal of Rheumatology.vol.14(3),pp.229-235,2019.

[63] van Heerde MJ, Buijs J, Hansen BE, de Waart M, van Eijck CH, Kazemier G, Pek CJ, Poley JW, Bruno MJ, Kuipers EJ, van Buuren HR. Serum level of Ca 19-9 increases ability of IgG4 test to distinguish patients with autoimmune pancreatitis from those with pancreatic carcinoma. Dig Dis Sci. 2014 Jun;59(6):1322-9
[64] M.-T.Chen, C.-K.Yao, C.-H.Chung, C.H.Shen, S.-H.Wang, C.-H.Wang, W.-C.Chien, C.-K.Peng, Increased risk of acute pancreatitis in patients with sj\&\#246;gren syndrome: A nationwide population-based cohort study. Journal of Medical Sciences.vol.40(1),pp.8$16,2020$.

[65] E.C.Ebert, Gastrointestinal and hepatic manifestations of Sjogren syndrome. Journal of clinical gastroenterology.vol.46(1),pp.2530,2012.

[66] Fukui T, Okazaki K, Yoshizawa H, Ohashi S, Tamaki H, Kawasaki K, Matsuura M, Asada M, Nakase H, Nakashima Y, Nishio A, Chiba T. A case of autoimmune pancreatitis associated with sclerosing cholangitis, retroperitoneal fibrosis and Sjögren's syndrome. Pancreatology. 2005;5(1):86-91 